\title{
Evidence and effects of a wave-driven nonlinear current in the equatorial electrojet
}

\author{
M. Oppenheim \\ Max-Planck-Institut für Aeronomie, Postfach 20, D-37191 Katlenburg-Lindau, Germany \\ Received: 20 May 1996 / Revised: 28 October 1996 / Accepted: 30 October 1996
}

\begin{abstract}
Ionospheric two-stream waves and gradientdrift waves nonlinearly drive a large-scale (D.C.) current in the $E$-region ionosphere. This current flows parallel to, and with a comparable magnitude to, the fundamental Pedersen current. Evidence for the existence and magnitude of wave-driven currents derives from a theoretical understanding of $E$-region waves, supported by a series of nonlinear 2D simulations of two-stream waves and by data collected by rocket instruments in the equatorial electrojet. Wave-driven currents will modify the large-scale dynamics of the equatorial electrojet during highly active periods. A simple model shows how a wave-driven current appreciably reduces the horizontally flowing electron current of the electrojet. This reduction may account for the observation that type-I radar echoes almost always have a Doppler velocity close to the acoustic speed, and also for the rocket observation that electrojet regions containing gradientdrift waves do not appear also to contain horizontally propagating two-stream waves. Additionally, a simple model of a gradient-drift instability shows that wavedriven currents can cause nonsinusoidal electric fields similar to those measured in situ.
\end{abstract}

\section{Introduction}

As early as the late middle ages, navigators observed that magnetic compass readings taken near the equator often varied by a few degrees in the day. In 1839, Friedrich Gauss speculated that these fluctuations resulted from the presence of large currents in the atmosphere. Balfour Stewart proposed in 1882 that these currents resulted from a solar-driven dynamo in an ionized region of the upper atmosphere which he called the ionosphere. At the beginning of this century, Schuster (1908) and Chapman (1919) developed a mathematical description of the dynamo which drives the equatorial electrojet.
Not long after the development of radar in the 1940s, Bowles et al. (1960) reported observing strong coherent radar echoes from the equatorial electrojet indicating the presence of plasma-density irregularities. A number of years later, Farley (1963) and Buneman (1963) applied linear kinetic and fluid theories to describe the origin of these echoes, now called the Farley-Buneman or two-stream instability. Both Maeda et al. (1963) and Simon (1963) extended this theory to describe a second $E$-region instability, the gradient-drift instability. However, linear theories cannot fully describe the behavior of these nonlinearly saturated waves.

This paper describes a nonlinear process resulting from $E$-region waves, its relative importance, and effects on a number of electrojet phenomena. We first described wave-driven currents in a letter (Oppenheim, 1996). This paper elaborates on the theory of wave-driven currents, extends the theory to describe longer wavelengths and discusses the effects of wave-driven currents on the largescale equatorial electrojet.

A wave-driven current results from two fundamental features of $E$-region plasma waves. First, electrons travel mostly perpendicular to the electric fields due to the geomagnetic field, while ions travel mostly parallel to the fields because ion-neutral collisions make magneticfield effects inconsequential. Second, gradient-drift and two-stream instabilities cause compressional waves where the plasma-density enhancements and the perturbed electric fields remain largely in phase. At the plasma-density maxima of the propagating wave fronts, electrons move perpendicular to the wave direction and the geomagnetic field. At the density minima, electrons move in the opposite direction with an equal velocity. However, more electrons exist at the maxima than at the minima causing a greater current in one direction than the other, resulting in a net (direct) current.

Evidence from rocket-based measurements and simulations show that the perturbed electric field of two-stream and gradient-drift waves has approximately the same order of magnitude as the polarization electric field. As we will show, this assumption makes the 
magnitude of the wave-driven current comparable to the electrojet's ion Pedersen current. This increase in current parallel to the polarization electric field may be approximated as a enhanced Pedersen conductivity. In an active electrojet (i.e., an electrojet containing waves) the effective Pedersen conductivity exceeds that of a quiet (i.e., wave-free) electrojet and will affect the largerscale behavior of $E$-region phenomena. For instance, in the equatorial electrojet, a wave-driven current works to reduce the polarization electric field that drives the waves which, in turn, drives the nonlinear current. Hence, this current may work as a saturation mechanism for the combined system of the wave and the polarization electric field.

This paper is organized as follows. First, we briefly discuss the literature relevant to wave-driven currents in the equatorial electrojet. Second, we describe the theory of a wave-driven current and its approximate magnitude. Third, we briefly describe the simulations that support our theoretical description of wave-driven currents. Fourth, we show how wave-driven currents may appreciably reduce the magnitude of the equatorial electrojet current and modify its distribution. Fifth, we demonstrate that wave-driven currents may cause nonsinusoidal electric fields within gradient-drift waves as measured by rockets.

\section{Background}

Since the first observations of coherent plasma structures in the ionosphere were made, hundreds of papers describing measurements and theories have been published. Numerous review papers, including Farley and Balsley (1973), Farley (1979, 1985), Fejer and Kelley (1980), and the book by Kelley (1989) survey this topic with varying degrees of detail. A recent review of theoretical developments can be found in Hamza and St.-Maurice (1995). Particle simulations of the twostream instability can be found in Janhunen (1994) and hybrid simulations are described in Oppenheim (1995) and Oppenheim et al. (1996). Recent fluid simulations of the gradient-drift instability have been carried out by Ronchi et al. (1991). Forbes (1981) reviews the physics of the equatorial electrojet while Richmond (1973a) and Forbes and Lindzen (1976) provide more detailed analyses.

Kudeki et al. (1985) used perturbation theory to evaluate the effects of nonlinear wave-driven currents on gradient-drift waves, explaining the measured direction and, roughly, the magnitude of the up-down asymmetry of secondary two-stream waves. They also argued that the current modifies the equatorial electrojet's vertical polarization electric field and its effective conductivity. We extend these arguments to the two-stream instability and show that the magnitude of the wave-driven current is comparable to the fundamental Pedersen current in the electrojet.

\section{Theory of wave-driven currents}

In the equatorial electrojet, two plasma instabilities result from electron $\vec{E} \times \vec{B}$ drifting. Both $E$-region instabilities create propagating compressional plasma density waves which drive nonlinear currents as illustrated by Fig. 1. The Farley-Buneman instability causes meter-scale plasma-density perturbations to grow when the electron drift speed exceeds the acoustic speed by an amount predicted by the following linear 1D relationship,

$\omega_{r}=\frac{k V_{d}}{\left(1+\Psi_{0}\right)}$,
$\omega_{i}=\frac{\omega_{r}^{2}-k^{2} C_{s}^{2}}{v_{i}\left(1+\Psi_{0}^{-1}\right)}$,

where $\omega_{r}$ is the real part of frequency and $\omega_{i}$ the imaginary part. The Earth's magnetic field, $\vec{B}_{0}$, is aligned with $\hat{y}$ and the electrojet's polarization electric field, $\vec{E}_{0}$ with $\hat{z}$. The linear traveling waves evolve as $\exp [i(\vec{k} \cdot \vec{x}-\omega t)]$. The electrons drift with velocity, $\vec{V}_{d}$ $=-E_{0} / B_{0} \hat{x}, k$ is the wavenumber of a wave propagating parallel to $V_{d}, \Psi_{0} \equiv v_{e} v_{i} /\left(\Omega_{e} \Omega_{i}\right), v_{i}$ and $v_{e}$ are the ion and electron collision frequencies, $\Omega_{i}$ and $\Omega_{e}$ are ion and electron cyclotron frequencies, $C_{s}$ is the acoustic velocity, $C_{s} \equiv \sqrt{\left[k_{b}\left(\gamma T_{e}+T_{e}\right) / m_{i}\right]}$, and $k_{b}$ is the Boltzmann constant. Further, to derive the dispersion equations, (1)-(2), we ignored kinetic effects and assumed $\omega_{i}$ small compared to $\omega_{r}$ and $v_{i}$ (Sudan et al., 1973). The two-stream growth-rate equation (2) predicts that the electron flow becomes linearly unstable and will experience wave growth when

$\omega_{r}^{2}>C_{s}^{2} k^{2} \Rightarrow V_{d}>C_{s}\left(1+\Psi_{0}\right)$.

The gradient-drift instability causes kilometer-scale wavelength plasma-density perturbations to grow when

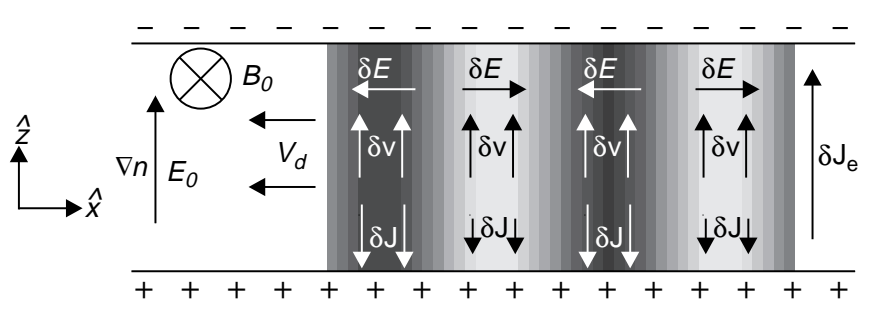

Fig. 1. Schematic representation of wave-driven currents in the equatorial electrojet. On the left of the figure, we show the vertical electrojet electric field, $E_{0}$, the geomagnetic field, $B_{0}$, the plasmadensity gradient, $\vec{\nabla} n$, and the electrojet electron drift direction, $V_{d}$. If $V_{d}$ exceeds a threshold then compressional plasma waves develop as shown by the varying shades of grey, darkest where the waves enhance the plasma density and lightest where they reduce it. At the density maxima and minima, we show the direction of the perturbed electric field, $\delta E$, the direction in which the electrons drift in response to $\delta E$, $\delta v$, and the resulting electron current, $\delta J=n \delta v$. This current is larger where the plasma density is enhanced than were it is reduced. On the right, we show the direction of the net, wave-driven, vertical electron current, $\delta J_{e}$. An identical mechanism generates wave-driven currents in the auroral electrojet when $\vec{\nabla} n=0, E_{0}$ is horizontal, and $B_{0}$ is vertical 
the plasma-density gradient points in the same direction as the polarization electric field. In the equatorial electrojet this typically occurs in the daytime. The following dispersion relation gives a simplified linear relationship between wave frequency and wave number for gradient-drift waves,

$\omega=\frac{\left(k+i k_{0}\right) V_{d}}{\left(1+\Psi_{0}^{-1}\right)\left(1+k_{0}^{2} / k^{2}\right)}$,

where $k_{0} \equiv\left[\kappa_{i}\left(1+\Psi_{0}\right) L\right]^{-1}$ and $L$ is the plasma-density gradient length, $L \equiv\left[\vec{\nabla} n / n_{0}\right]^{-1}$ (Kudeki et al., 1982; Rogister, 1972). See Fejer et al. (1975) for a more complete dispersion relation. For $k \gg k_{0}$, Eq. (4) reverts to the dispersion relationship for the Farley-Buneman instability, ignoring diffusive effects (i.e., $C_{s}=0$ ).

The 1D linear theories of both the Farley-Buneman and gradient-drift instabilities predict that any perturbation in the plasma density, $\delta n$, will grow exponentially if a large enough $\left|V_{d}\right|$ and/or gradient in the background plasma density, $n_{0}$, exists. A perturbed horizontal electric field, $\delta E_{x}$, also grows exponentially with the following relationship to $\delta n$,

$\delta E_{x}=\left[\frac{-E_{0}\left[1-i \kappa_{e} /(L k)\right]}{\kappa_{i}\left(1+\Psi_{0}\right)\left[1+\kappa_{e}^{2} /(L k)^{2}\right]}+i k \frac{k_{b} T_{e}}{e}\right] \frac{\delta n}{n_{0}}$,

where $\kappa_{i} \equiv \Omega_{i} / v_{i}$ and $\kappa_{e} \equiv \Omega_{e} / v_{e}$ (Kudeki et al., 1985). The relationship between $\delta n$ and $\delta E_{x}$ derives from a combination of the quasi-neutrality assumption, $\vec{\nabla} \cdot \vec{J}=0$, with the inertialess electron momentum equation and the ion continuity and momentum equations. However, Eq. (5) only approximates the relationship between $\delta E$ and $\delta n$ well for $E$-region waves longer than a meter but shorter than the kilometer-scale plasma-density gradient. For shorter waves, kinetic effects modify this relationship. For longer waves, the relationship between $\delta E$ and $\delta n$ incorporates a number of additional terms reflecting the magnitude of the plasma-density gradient. A relationship valid for longer wavelengths contains many powers of $k_{0} / k$ and, while the added complexity does not eliminate the wavedriven current, it does make it more difficult to see the fundamental physics of the current.

The term containing the electron temperature, $T_{e}$, results from electron diffusion which we shall neglect: it tends to be small and the resulting electric field is $90^{\circ}$ out of phase with the density wave and does not result in a net electron current when averaged over an entire wave. We shall also neglect the $i \kappa_{e} /(L k)$ component, because it, too, is out of phase with the density wave.

Electrons respond to this perturbed electric field by $\delta \vec{E} \times \vec{B}$ drifting parallel to $\vec{E}_{0}$ on the wave crests and opposite to $\vec{E}_{0}$ in the wave valleys with the following velocity,

$\delta \vec{v}_{e}=\frac{\delta E_{x} \hat{x} \times \vec{B}_{0}}{B_{0}^{2}}=-\frac{E_{0}}{B_{0}} \frac{\delta n}{n_{0}} \frac{1}{\kappa_{i}\left(1+\Psi_{0}\right)\left[1+\kappa_{e}^{2} /(L K)^{2}\right]} \hat{z}$.

Hence, the electrons travel in the $+\hat{z}$ direction with the same velocity at the maxima of the density enhance- ments as they travel in the $-\hat{z}$ direction at the minima. However, at the maxima the density exceeds that at the minima, so more electrons drift in the $+\hat{z}$ direction than in the $-\hat{z}$ direction. The net electron current averaged over a single wave is

$$
\begin{aligned}
\left\langle J_{e z}\right\rangle & =\frac{e}{\kappa_{i}\left(1+\Psi_{0}\right)\left[1+\kappa_{e}^{2} /(L k)^{2}\right]} \frac{E_{0}}{B_{0}} \frac{|\delta n|^{2}}{n_{0}} \int_{0}^{\lambda} \sin ^{2}(k x) \frac{d x}{\lambda} \\
& =\frac{e\left(E_{0} / B_{0}\right)}{\kappa_{i}\left(1+\Psi_{0}\right)\left[1+\kappa_{e}^{2} /(L k)^{2}\right]} \frac{|\delta n|^{2}}{n_{0}}\left(\frac{1}{2}\right)
\end{aligned}
$$

where $\lambda=2 \pi / k$ is the wavelength. The presence of $k$ in the $\kappa_{e}^{2}(L k)^{2}$ term causes a reduction in the size of the nonlinear current as the wavelength approaches the plasma gradient length, $L$. To obtain the total wavedriven current in the electrojet, one should sum the current in all modes.

How does the magnitude of a wave-driven current compare to other currents in the $E$ region? This depends on the unknown, nonlinearly determined quantity, $|\delta n|$. For two-stream waves in the equatorial electrojet, evidence from in situ experiments and simulations suggest that the average $\delta n / n_{0}$ is 0 to 10 percent and magnitude of the perturbed electric field, $\langle\delta E\rangle$, is similar to $E_{0}$ (see Pfaff et al., 1987b; Oppenheim et al., 1996). For gradient-drift waves, the $\langle\delta E\rangle$ is more difficult to estimate, though it has been measured to reach approximately $15 \mathrm{mV} / \mathrm{m}$, corresponding to the largest measured values of $E_{0}$ (Pfaff et al., 1987a; Prakash et al., 1974). Using Eqs. (5) and (7) and $\langle\delta E\rangle=E_{0}$, we can estimate the magnitude of the wave-driven current as

$\left\langle J_{e z}\right\rangle=e n_{0} \frac{E_{0}}{B_{0}} \frac{\kappa_{i}\left(1+\Psi_{0}\right)\left[1+\kappa_{e}^{2} /(L k)^{2}\right]}{2}$.

For wavelengths considerably shorter than $L$, both $\kappa_{i}$ and $\Psi_{0}$ are of the order of 0.1 . Hence, the nonlinear electron current is approximately $5 \%$ of the drift current. However, its importance derives from the fact that it flows perpendicular to the electron drift current and with the same order of magnitude as the ion-Pedersen current.

Using $J_{i p} \approx E_{0} n_{0} e^{2} /\left(m_{i} v_{i}\right) \hat{z}$ to approximate the ionPedersen current and Eq. (8) to estimate the wave-driven current magnitude, we obtain the ratio,

$\frac{\left\langle J_{e z}\right\rangle}{J_{i p}}=\frac{\left(1+\Psi_{0}\right)\left[1+\kappa_{e}^{2} /(L k)^{2}\right]}{2} \sim 1$.

Hence, the wave-driven current has a similar magnitude to the ion-Pedersen current. Since the ion-Pedersen current plays an important role in $E$-region dynamics, so must the wave-driven current.

\section{Two-stream wave simulations}

In nature, the nonlinear behavior of electrojet waves may disrupt the phase relationship between $\delta E$ and $\delta n$, greatly reducing or eliminating the wave-driven currents. Our simulations of the Farley-Buneman instability do not show such a disruption. This $2 \mathrm{D}$ simulation 


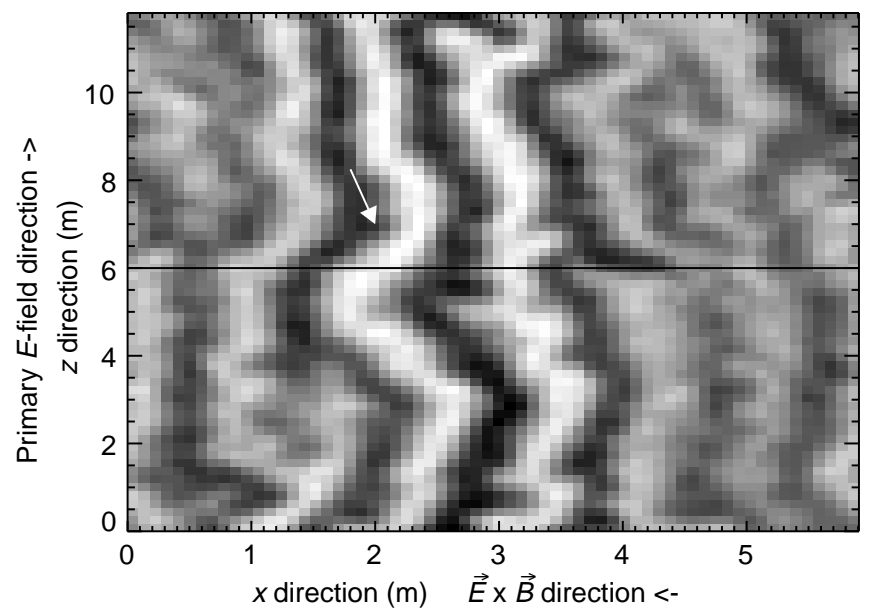

Fig. 2. Perturbed density of waves in a saturated state from a typical simulation of two-stream waves in a saturated state. The primary wave front propagates leftward. As the primary waves travel leftward, the crests of secondary waves travel downward along the wave fronts as indicated by the bold arrow. Likewise, minima travel not only leftward but also upward. Vertical currents are calculated by summing ion and electron flows across the horizontal line shown

models the plane perpendicular to the geomagnetic field with particle-in-cell (PIC) ions and fluid electrons. Oppenheim et al. (1996) describes the simulation technique and the fundamental results. Oppenheim and Otani (1996) shows that these simulations reproduce many of the observed features of ionospheric twostream waves by comparing the simulation spectra with those observed by radar and rockets. All these simulations show wave-driven currents of appreciable magnitude and flowing in the direction predicted by the theory just presented. Figure 2 shows a typical plasmadensity distribution of two-stream waves in a saturated state as calculated by this hybrid code.

We calculated the vertical currents flowing in the simulations by totaling the electron and ion flows crossing an imaginary horizontal line. Table 1 shows the parameters used by two simulations and Fig. 3 shows the various vertical currents generated by these simulations. The first simulation models conditions

Table 1. Parameters of baseline Farley-Buneman simulation

\begin{tabular}{lll}
\hline Physical parameters & Sim. 1 & Sim. 2 \\
\hline Magnetic field $\left(\vec{B}_{0}\right.$ Gauss $)$ & 0.25 & 0.25 \\
$\vec{E}_{0}$ field $\left(E_{0 y}\right.$ Vlm $)$ & -0.015 & -0.0145 \\
Avg. ion density $\left(n_{0} \mathrm{~m}^{-3}\right)$ & $10^{11}$ & $2 \times 10^{11}$ \\
neutral density $\left(n_{n} \mathrm{~m}^{-3}\right)$ & $6 \times 10^{18}$ & $2 \times 10^{18}$ \\
Initial Temp. $\left(T^{\circ} \mathrm{K}\right)$ & 208 & 250 \\
Effective ion mass $\left(m_{i} k g\right)$ & $4.6 \times 10^{-26}$ & $5 \times 10^{-26}$ \\
$\mathrm{e}^{-}-n$ col. freq. $\left(v_{e} s^{-1}\right)$ & $4.0 \times 10^{4}$ & $1.7 \times 10^{4}$ \\
Ion- $n$ col. freq. $\left(v_{i} s^{-1}\right)$ & $2.8 \times 10^{3}$ & 950 \\
\hline Simulation parameters & & \\
\hline Total box length $(m)$ & $3 \times 6$ & $6 \times 12$ \\
Grid resolution & $64 \times 64$ & $64 \times 64$ \\
Time-step $(s)$ & $1 \times 10^{-5}$ & $1 \times 10^{-5}$ \\
Number of ions & 921600 & 2073600 \\
\hline
\end{tabular}
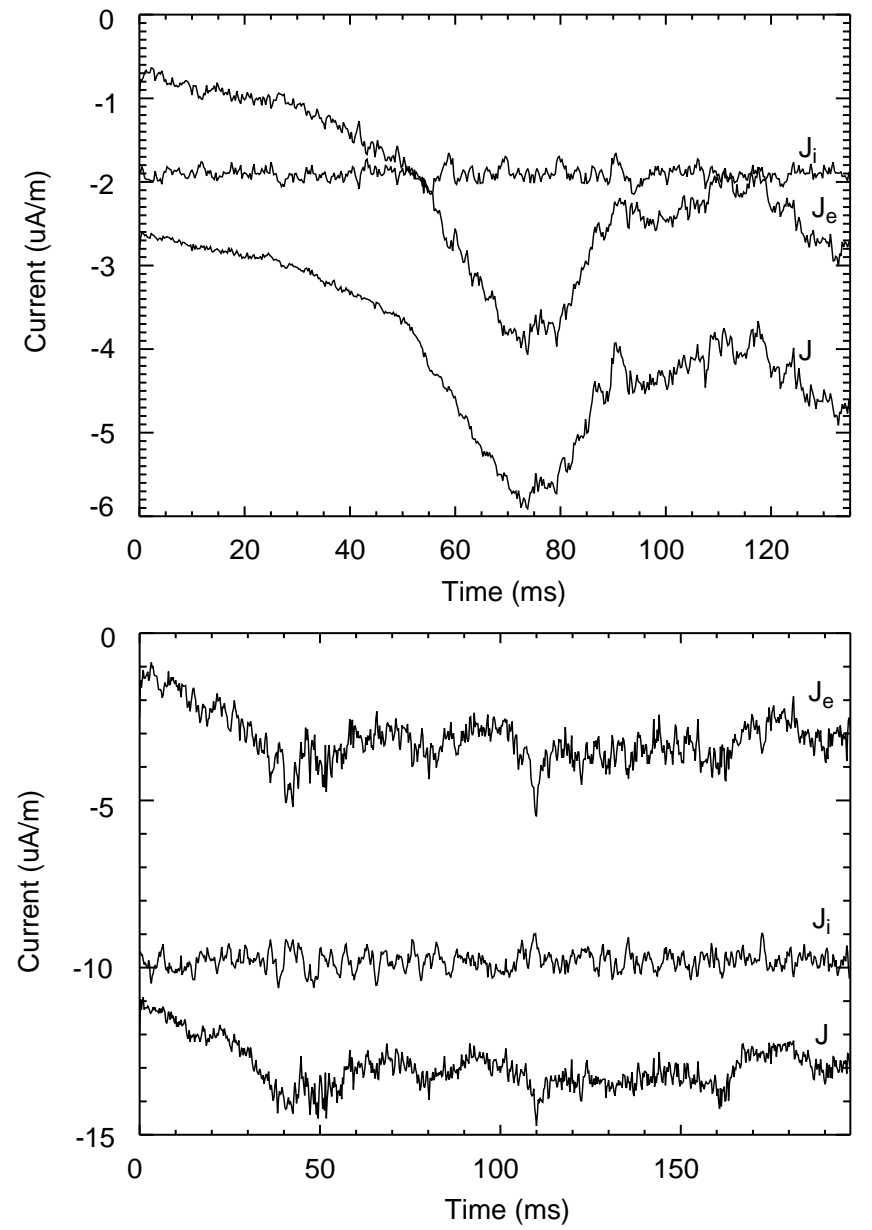

Fig. 3. Vertical currents versus time from two Farley-Buneman instability simulations with the parameters shown in Table 1 . The upper figure derives from simulation 1 while the lower from simulation 2 . Both show the ion current, $J_{i}$, the electron current, $J_{e}$, and the total vertical current, $J . J_{i}$ shows the ion-Pederson current, largely unmodified by the wave growth. $J_{e}$ starts with a small electronPedersen current and develops a wave-driven current as the instability grows

found at the bottom of the equatorial electrojet, while the second models the middle to upper portion of the electrojet. The current in both simulations flows in the same direction as predicted by Eq. (9). In the first case, the wave-driven current exceeds the ion-Pedersen current in magnitude while, in the second case, the wave-driven current is about one-third the ion-Pedersen current.

Additionally, all our two-stream simulations show plasma-density perturbations traveling perpendicular to the wave direction as indicated in Fig. 2. These additional traveling waves may result from a secondary FarleyBuneman-type instability driven by the nonlinear $\vec{E} \times \vec{B}$ drifting electrons. They differ from the standard FarleyBuneman instability because they appear on the extrema of primary two-stream waves. Oppenheim et al. (1996) develops this idea further. Motion pictures show the dynamic behavior of our simulation far better than still images and may be viewed directly with WWW browser at the addresses http://www.ee.cornell.edu/ $\sim$ meers and http://www.mpae.gwdg.de/publications/Oppenheim 


\section{Equatorial electrojet model}

When two-steam and/or gradient-drift waves exist within the equatorial electrojet, wave-driven currents will play an important role in determining the current magnitude and distribution. A detailed model of the equatorial electrojet depends on numerous complex 3D factors including neutral winds, magnetic-field geometry, and the plasma distribution and composition (Forbes, 1981; Richmond, 1973a, b). Such a model is beyond the scope of this paper. Our objective is to build the simplest possible model of the equatorial electrojet and to illustrate how a wave-driven current modifies the electrojet current. Further, we discuss how a more complete model may be studied.

Using Maxwell's equations and the Lorenz force equation without particle inertia, the relationship between the local current, the electric field, and the neutral-wind velocity is

$$
\begin{aligned}
& \vec{J}=\hat{\sigma}(\vec{E}+\vec{U} \times \vec{B}), \\
& \hat{\sigma} \equiv\left(\begin{array}{lll}
\sigma_{x x} & \sigma_{x y} & \sigma_{x z} \\
\sigma_{y x} & \sigma_{y y} & \sigma_{y z} \\
\sigma_{z x} & \sigma_{z y} & \sigma_{z z}
\end{array}\right), \\
& \sigma_{x x}=\sigma_{P} \\
& \sigma_{x y}=-\sigma_{y x}=-\sigma_{H} \sin I, \\
& \sigma_{x z}=-\sigma_{z x}=\sigma_{H} \cos I \\
& \sigma_{y y}=\sigma_{P} \sin ^{2} I+\sigma_{0} \cos ^{2} I, \\
& \sigma_{y z}=\sigma_{z y}=\left(\sigma_{0}-\sigma_{P}\right) \sin I \cos I, \\
& \sigma_{z z}=\sigma_{P} \cos ^{2} I+\sigma_{0} \sin ^{2} I,
\end{aligned}
$$

where the parallel, $\sigma_{0}$, Pedersen, $\sigma_{P}$, and Hall, $\sigma_{H}$, conductivities are defined by

$$
\begin{aligned}
\sigma_{0} & \equiv \sum_{m=1}^{M} \frac{N_{m} q_{m}}{B} \frac{\Omega_{m}}{v_{m}} \\
\sigma_{P} & \equiv \sum_{m=1}^{M} \frac{N_{m} q_{m}}{B} \frac{v_{m} \Omega_{m}}{\Omega_{m}^{2}+v_{m}^{2}} \\
\sigma_{H} & \equiv \sum_{m=1}^{M} \frac{N_{m} q_{m}}{B} \frac{\Omega_{m}^{2}}{\Omega_{m}^{2}+v_{m}^{2}}
\end{aligned}
$$

(Forbes, 1981). Also, $I$ is the magnetic dip angle (the angle the magnetic field line makes with respect to horizontal), $\vec{U}$ is the neutral-wind velocity, $M$ is the number of species present in the plasma; $N_{m}$ is the number density, $q_{m}$ is the charge, $v_{m}$ is the collision rate, and $\Omega_{m}$ is the cyclotron frequency of species $m$. Assuming quasi-neutrality, $\vec{\nabla} \cdot \vec{J}=0$, allows one to produce a single equation for the electric potential in the equatorial electrojet,

$$
\vec{\nabla} \cdot(\hat{\sigma} \vec{\nabla} \phi)=\vec{\nabla} \cdot \hat{\sigma}(\vec{U} \times \vec{B})
$$

This 3D partial differential equation governs the largescale behavior of the equatorial electrojet. By choosing appropriate boundary conditions and neutral-wind behavior, this equation predicts the strength and distribution of the electrojet. By neglecting particle inertia and diffusion, Eq. (21) does not include the physics which drives two-stream waves.

To generate the simplest possible model of the electrojet which incorporates the effects of a wavedriven current, we assume a slab model of the $E$ region. This reduces Eq. (21) to a $1 \mathrm{D}$ equation by requiring homogeneity in both the $\hat{x}$ and $\hat{y}$ directions,

$E_{z}=-\left(\sigma_{H} / \sigma_{P}\right)\left(E_{x}+B_{0} U_{x}\right)$.

To incorporate a wave-driven current into this system as an additional phenomenological current, we add $J_{n l}$ to Eq. (10). Requiring quasi-neutrality modifies the full 3D governing equation from (21) to

$$
\vec{\nabla} \cdot(\hat{\sigma} \vec{\nabla} \phi)=\vec{\nabla} \cdot \hat{\sigma}(\vec{U} \times \vec{B})+\vec{\nabla} \cdot \vec{J}_{n l}
$$

and the simplified slab-model equation becomes

$E_{z}=-\left(\sigma_{H} / \sigma_{P}\right)\left(E_{x}+B_{0} U_{x}\right)-J_{n l z} / \sigma_{P}$.

Using Eq. (24) and making a number of simple assumptions about the configuration of the electrojet, we can compare the resulting vertical current distribution with and without a subsequent nonlinear current. First, we must decide on the magnitude of $E_{x}$ and $U_{x}$. In a $3 \mathrm{D}$ electrojet model, $E_{x}$ and $U_{x}$ arise from the tidal winds, the spatially and temporally variable conductivities, and the total current path. However, our simple 1D model includes none of these features, so we must assume values for $E_{x}$ and $U_{x}$. Ideally, we could use values from data gathered by rocket instruments but, in an active electrojet, these small values have proven nearly impossible to measure. Instead, we follow the example of Richmond (1973b) and solve for typical values for $E_{x}$ and $U_{x}$ by introducing known electrojet current profiles into Eqs. (10) and (22). This analysis enables us to choose $E_{x}=1 \mathrm{mV}$ and $U_{x}=0$.

Using typical midday electrojet values for the plasma density and composition, we determine $\sigma_{H}$ and $\sigma_{P}$ from Eqs. (19) and (20) (Johnson, 1965). The nonlinear current, $J_{n l z}$, is made to equal the ion-Pedersen current times $\left(1+\Psi_{0}\right) / 4$, which is half the value suggested by Eq. (9) but comparable to the current generated by the second simulation shown in Fig. 3 with the parameters given in Table 1.

Finally, we assume that $E_{x}, U_{x}, \sigma_{H}$, and $\sigma_{P}$ remain constant despite the development of waves. These assumptions enable one to solve for the modified vertical electric field as

$E_{z}=-\frac{\left(\sigma_{h} / \sigma_{P}\right)\left(E_{x}+B_{0} U_{x}\right)}{1+\sigma_{P i} / \sigma_{P}\left(1+\Psi_{0}\right) / 4}$

where $\sigma_{P i}$ is the ion-Pederson conductivity, which is usually only slightly less than $\sigma_{P}$. The resulting electrojet current is shown in Fig. 4.

When wave-driven currents arise from two-stream waves, the solution for the electrojet current has three 




Fig. 4. Electrojet current density as a function of height. The dashed line shows the current without nonlinear effects, the solid line shows the current including effects of a two-stream wave-driven nonlinear current, the dash-dotted line shows the current including effects of a gradient-drift (GD) wave-driven current and the dotted line shows the minimum current necessary for initiating two-stream waves and the resulting wave-driven current

distinct regions. First, the top and bottom of the electrojet contain no waves, and so, the nonlinearly modified electrojet current (solid line) matches the unmodified current (dashed line). Second, in the upper and lower part of the electrojet which contains waves, the nonlinear current causes the horizontal electron velocity to fall below the threshold for driving waves. In these regions we expect the electrojet current to diminish to a value fairly close to the threshold current (dotted line). In the center of the wave-containing region, the wave-driven current reduces the electrojet current to a magnitude lying below the unmodified electrojet but above the current representing the instability threshold. In our simple model this intermediate current derives from Eq. (25).

Regions of the electrojet where the nonlinear current reduces the electric field sufficiently to make the waves linear stable or damped present an interesting question. In the 1D model, if we increase the wave-driven current magnitude to half the ion-Pedersen current and reduce the driving electric field $E_{x}$ by $20 \%$, the region where the wave-driven current stabilizes the two-stream instability encompasses the entire wave-containing region of the electrojet. Is there an equilibrium point where the nonlinear current forces the polarization electric field to fix at the wave threshold, $E_{0 z}=B C_{s}\left(1+\Psi_{0}\right)$ ? Or, does the system oscillate around the threshold? Unfortunately, it is currently difficult to model numerically two-stream waves when the driving electric field only slightly exceeds the threshold. Hybrid and particle codes require the wave amplitude to exceed the amplitude of the particle noise, a condition met when the driving electric field exceeds the threshold by more than a few percent.

When gradient-drift waves drive the nonlinear current, the threshold polarization electric field for initiating waves is very low. Therefore, the wave-driven current reduces the electrojet current to a magnitude below that of the unmodified electrojet as derived from Eq. (25).

The results from such a highly simplified model probably cannot be compared directly to those of the actual equatorial electrojet. An improved model should probably include a 2 - or $3 \mathrm{D}$ model of the electrojet. Second, the nonlinear current magnitude should be evaluated through simulations. This will require developing techniques of studying marginally stable $E$-region waves.

\section{Gradient-drift wave simulation}

The electric fields measured by rockets passing through gradient-drift waves often appear as irregular square waves (Pfaff et al., 1987a). We show that wave-driven electron currents can cause these squared-off electric fields through a two-step process. First, the perturbed electric field of a gradient-drift wave must exceed the threshold necessary to initiate two-stream waves (Sudan et al., 1973). Second, these secondary two-stream waves generate wave-driven electron currents which modify the original gradient-drift waves.

To study the effect of a wave-driven current on a gradient-drift wave, we developed a 1D numerical model. The long-wavelength, gradient-drift instability behavior was modeled with inertialess ions and electrons, no temperature effects, and no nonlinear components except for a wave-driven current. The nonlinear current was added to the Pedersen current wherever the electric field of the gradient-drift waves exceeded the threshold necessary to drive two-stream instabilities.

Starting with a linearized form of the ion and electron continuity equations,

$\frac{\partial s}{\partial t}=\frac{1}{\Psi_{0}} \frac{\partial V_{e x}}{\partial x}$

$\frac{\partial s}{\partial t}=-\frac{\partial V_{e x}}{\partial x}-V_{d} \frac{\partial s}{\partial x}-\frac{\kappa_{e}}{L} V_{e x}-\frac{1}{e n_{0}} \frac{\partial J_{n l x}}{\partial x}$,

where $s$ is the $\log$ of the normalized plasma density, $s \equiv \ln \left(n / n_{0}\right)$. By adding and subtracting these equations and then Fourier transforming the resulting equations from $\hat{x}$-space (real space) into $k$-space, we obtain an equation governing the time evolution of the gradientdrift instability,

$\frac{\partial s}{\partial t}=\left(s-\frac{J_{n l}}{e n_{0} V_{d}}\right) \frac{-i k V_{d}}{\left(\Psi_{0}+1\right)+\Psi_{0} \kappa_{e} /(i k L)}$,

where all spatially varying parameters and variables are required to be periodic.

We approximated a solution to Eq. (28) numerically with the fourth-order Runge-Kutta method (Press et al., 1988). After calculating the density $s$, at each time-step, the program calculated the electric field and Fourier transformed it to obtain a real-space value for the perturbed horizontal electric field. Wherever the electric field exceeded the threshold necessary to drive two- 

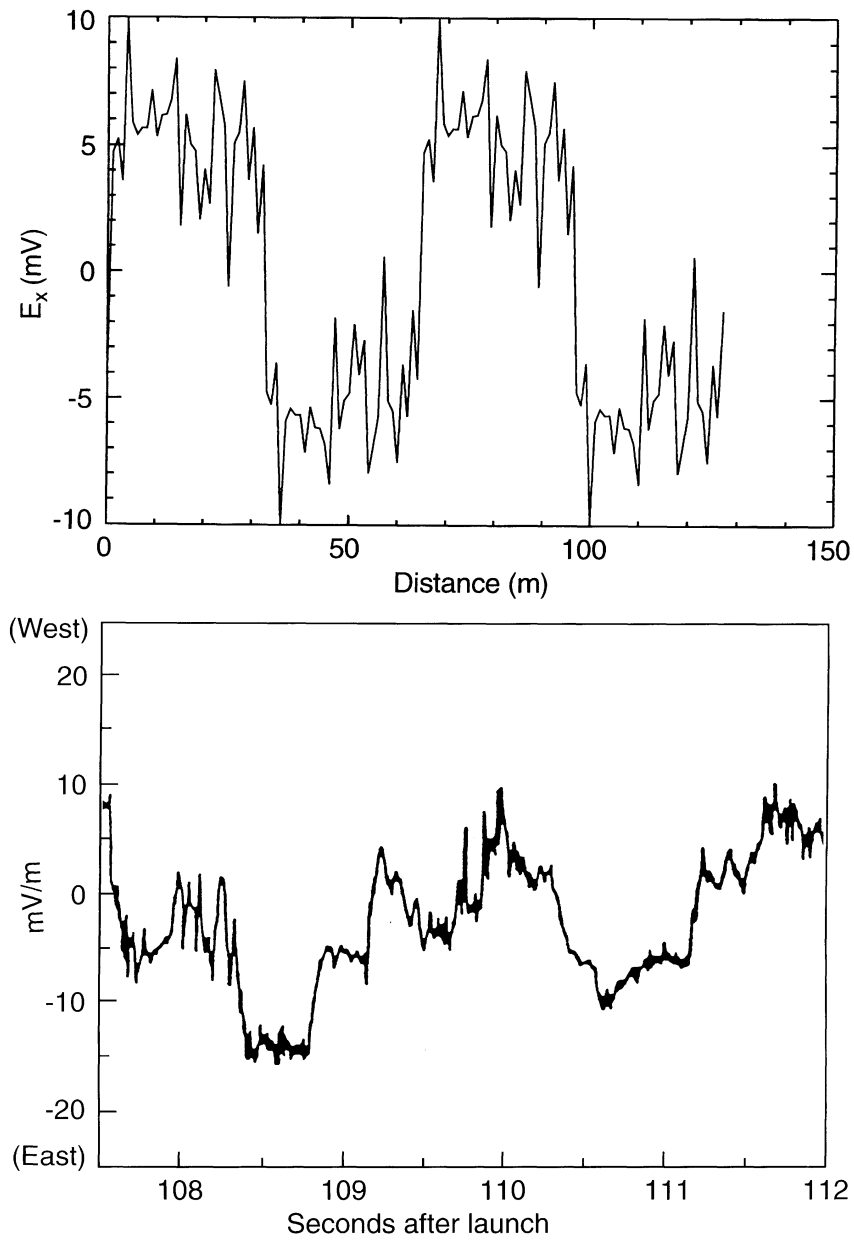

Fig. 5. The electric field from a $1 \mathrm{D}$ simulated gradient-drift wave system modified by secondary two-stream waves driving a nonlinear current (top). The measured fields by Pfaff et al. (1987b) (bottom)

stream waves, the wave-driven current, $J_{n l}$, was made equal to half the ion-Pedersen current.

We began the simulation with a small gradient-drift wave which grew exponentially until its amplitude reached the threshold for two-stream waves. After a number of cycle times the perturbed electric field appeared similar to the measured fields show in Figs. 4-9 of Plaff et al. (1987a), as shown here in Fig. 5. The addition of the wave-driven current also had the effect of dramatically showing gradient-drift wave growth.

Neither the squaring of the waves nor the slowing of the growth is surprising, given the simple model we used. It is almost inevitable that when one adds an effect which works to reduce the perturbed electric field at the crest of a wave, that wave will become somewhat square. Also, reducing the perturbed electric fields and the perturbed plasma densities at the extrema should disrupt the exponential growth. While one might enhance a similar $1 \mathrm{D}$ model by adding diffusion, recombination, and (or) ion inertia, a more important enhancement is to add the second dimension perpendicular to $B$. While such a 2D simulation exceeds the current computational capacity of PIC or hybrid codes, a fluid code should be able to model this system. Such a code could distinguish between various competing saturation mechanisms for gradient-drift waves.

\section{Discussion and conclusions}

We have shown that a large-scale, wave-driven current results from the nonlinear dynamics of two-stream and gradient-drift waves. During quiet periods in the $E$ region, when no waves exist, only Pedersen currents flow parallel to the electric field. During active periods, when waves exist, a wave-driven current will increase the total current parallel to the polarization electric field. If we make the well-justified assumption that the perturbed electric field is, on average, the same order of magnitude as the electrojet's polarization field, then the resulting wave-driven current will have approximately the same size and direction as the ion-Pedersen current. The increase in current may be modeled as a drop in electrojet resistance. However, adding an explicit electron current, as done in our 1D models of the equatorial electrojet and gradient-drift wave is a more accurate method.

Wave-driven currents will reduce the equatorial electrojet current as illustrated by the simple model described in the present paper. In an electrojet containing only two-stream waves, where the polarization electric field only slightly exceeds the threshold necessary to initiate waves, the wave-driven current will hold the polarization electric field to a value close to the threshold. If the polarization electric field greatly exceeds the threshold, then it will be reduced, in some regions of the electrojet, to a value close to the threshold and, in other regions, to a value between the threshold and the field expected if no wave-driven current existed. Linear theory predicts that when the polarization electric field is close to its threshold value for initiating two-stream waves then these waves travel at the acoustic velocity. This may account for the observation that two-stream waves travel at speeds close the acoustic velocity.

In an electrojet containing mostly gradient-drift waves, we expect wave-driven currents to reduce the polarization electric field. The rocket observation by Pfaff et al. (1987a), that equatorial electrojet regions containing gradient-drift waves do not appear also to contain horizontally propagating two-stream waves, may result from wave-driven currents working to reduce the polarization electric field to a point below the threshold required to initiate two-stream waves. Further, since the polarization electric field provides the energy for the both the gradient-drift and twostream instability, we expect wave-driven currents to play a role in saturating these instabilities.

Wave-driven currents affect the behavior of gradientdrift waves. Kudeki et al. (1985) used a perturbation analysis of wave-driven currents to account for the updown asymmetry of secondary two-stream waves. They also suggested that the current modifies the equatorial electrojet's vertical polarization electric field and its effective conductivity. We have built a simple model of 
the equatorial electrojet and estimated the magnitude of the effect of wave-driven currents on it for both gradient-drift and two-stream waves.

When the perturbed horizontal electric field of gradient-drift waves exceeds the threshold necessary to drive secondary two-stream waves, then the resulting wave-driven currents will modify the driving gradientdrift waves. A simple 1D simulation shows that the resulting electric field reproduces the electric-field measurements made by rockets.

Wave-driven currents are not restricted to the equatorial electrojet. In the auroral electrojet, large electric fields result from a complex interaction between the solar wind and the magnetosphere. Currents generated high above the ionosphere propagate along the Earth's magnetic field lines until they reach the $E$ region, the lowest altitude where substantial currents flow across magnetic field lines. When the resulting polarization electric field becomes large enough to drive waves, wave-driven currents will carry current across the magnetic field lines, effectively dropping the $E$-region resistance. The repercussions of this change may very well propagate back into the magnetosphere and affect the dynamics of this global-scale dynamo.

Electron temperatures in the auroral electrojet have been observed substantially to exceed ion temperatures (Schlegel and St.-Maurice, 1981; Wickwar et al., 1981). Wave-driven currents may play a role in electron heating. However, heating by the oscillations of the electrons perpendicular to the wave direction (see Fig. 1) should include and exceed the effect of heating by the wave-driven current. One may use the assumption that the perturbed electric field is similar in magnitude to the polarization electric field to estimate the magnitude of the electron heating.

Rockets should be able to infer the existence of wavedriven currents by averaging the electron currents across many waves, either with current monitors or with magnetometers. If such direct measurements are too difficult, they should be able to measure both the perturbed electric field and the plasma density at many points in a wave and calculate the current driven by $\vec{E} \times \vec{B}$ drifting electrons. Determining the magnitude of a wave-driven current, given known ionospheric conditions, should greatly increase our understanding of electrojet physics.

A fundamental question about $E$-region instabilities has been, "Do they play an appreciable role in the dynamics of the electrojet or are they interesting only because our radars and rockets detect them?" We believe that, because of wave-driven currents, waves in the electrojet are not simply measurable evidence of strong currents, but rather play an integral role in modifying the fundamental nature of the electrojet.

Acknowledgments. The author gratefully acknowledges the help and insights of D. Farley, T. Hagfors, N. Otani, K. Schlegel, C. Ronchi, C. Seyler, and R. Mason. This work was mostly supported by the Max-Planck-Gesellschaft and partially supported by funding from the IGPP program at Los Alamos (subcontract 9XA3-Q7557-1), NASA (NAGW-3010) and NSF (PYI ATM9158072).
Topical Editor D. Alcaydè thanks P. Janhunen and another referee for their help in evaluating this paper.

\section{References}

Bowles, K. L., R. Cohen, G. R. Ochs, and B. B. Balsley, Radar echoes from field-aligned ionization above the magnetic equator and their resemblence to auroral echoes, J. Geophys. Res., 65, 1853, 1960.

Buneman, O., Excitation of field aligned sound waves by electron streams, Phys. Rev. Lett., 10, 285, 1963.

Chapman, S., The solar and lunar diurnal variations of terrestrial magnetism, Philos. Trans. R. Soc. London, Ser. A, 218, 1-118, 1919.

Farley, D. T., A plasma instability resulting in field-aligned irregularities in the ionosphere, J. Geophys. Res., 68, 6083, 1963.

Farley, D. T., The ionospheric plasma, in Solar System Plasma Physics, Eds. C. F. Kennel, L. J. Lanzerotti, and E. N. Parker, North-Holland, New York, pp 211-270, 1979.

Farley, D. T., Theory of equatorial electrojet plasma waves: new developments and current status, J. Atmos. Terr. Phys., 47, 729, 1985.

Farley, D. T., and B. B. Balsley, Instabilities in the equatorial electrojet, J. Geophys. Res., 78, 227, 1973.

Fejer, B. G., and M. C. Kelley, Ionospheric irregularities, Rev. Geophys. Space Phys., 18, 401, 1980.

Fejer, B. G., D. T. Farley, B. B. Balsley, and R. F. Woodman, Vertical structure of the vhf backscattering region in the equatorial electrojet and the gradient drift instability, $J$. Geophys. Res., 80, 1313, 1975.

Forbes, J. M., The equatorial electrojet, Rev. Geophys. Space Phys., 19, 469, 1981.

Forbes, J. M., and R. S. Lindzen, Atmospheric solar tides and their electrodynamic effects. II. The equatorial electrojet, J. Atmos. Terr. Phys., 38, 911, 1976.

Hamza, A. M., and J. P. St.-Maurice, A fully self-consistent fluid theory of anomalous transport in Farley-Buneman turbulence, J. Geophys. Res., 100, 9653, 1995.

Janhunen, P., Perpendicular particle simulation of the E-region Farley-Buneman instability, J. Geophys. Res., 99, 11461, 1994.

Johnson, F. S., Satellite Environment Handbook, Stanford University Press, Stanford, Calif., 1965.

Kelley, M. C., The Earth's Ionosphere, Academic Press, San Diego, Calif., 1989.

Kudeki, E., D. T. Farley, and B. G. Fejer, Long-wavelength irregularities in the equatorial electrojet, Geophys. Res. Lett., 9, 684, 1982.

Kudeki, E., D. T. Farley, and B. G. Fejer, Theory of spectral asymmetries and nonlinear currents in the equatorial electrojet, J. Geophys. Res., 90, 429, 1985.

Maeda, K., T. Tsuda, and H. Maeda, Theoretical interpretation of the equatorial sporadic E layers, Phys. Rev. Lett., 11, 406, 1963.

Oppenheim, M. M., Nonlinear simulations and theory of the Farley-Buneman instability in the E-region ionosphere, $P h . D$. thesis, Cornell University, Ithaca, NY., 1995.

Oppenheim, M. M., A wave-driven nonlinear current in the Eregion ionosphere, Geophys. Res. Lett., 23, in-review, 1996.

Oppenheim, M. M., and N. F. Otani, Spectral characteristics of the Farley-Buneman instability: simulations versus observations, $J$. Geophys. Res., 101, in-press, 1996.

Oppenheim, M. M., N. F. Otani, and C. Ronchi, Saturation of the Farley-Buneman instability via nonlinear electron $\vec{E} \times \vec{B}$ drifts, J. Geophys. Res., 101, in-press, 1996.

Pfaff, R. F., M. C. Kelley, E. Kudeki, B. G. Fejer, and K. Baker, Electric field and plasma density measurements in the strongly driven daytime equatorial electrojet, 1, The unstable layer and gradient drift waves, J. Geophys. Res., 92, 13578, 1987a.

Pfaff, R. F., M. C. Kelley, E. Kudeki, B. G. Fejer, and K. Baker, Electric field and plasma density measurements in the strongly 
M. Oppenheim: Evidence and effects of a wave-driven nonlinear current in the equatorial electrojet

driven daytime equatorial electrojet, 2, Two-stream waves, $J$. Geophys. Res., 92 13597, 1987b.

Prakash, S., C. L. Jain, B. B. Balsley, and R. A. Greenwald, Evidence of two types of electron density irregularities in the electrojet over Thumba, India, J. Geophys. Res., 79, 4334, 1974.

Press, W., B. Flannery, S. Teukolsky, and W. Vetterling, Numerical Recipes in C, Cambridge University Press, New York, 1988.

Richmond, A. D., Equatorial electrojet, I. development of a model including winds and instabilities, J. Atmos. Terr. Phys., 35, 1083, 1973a.

Richmond, A. D., Equatorial electrojet, II. use of the model to study the equatorial ionosphere, J. Atmos. Terr. Phys., 35, 1103, $1973 b$.

Rogister, A., Nonlinear theory of cross field instability with application to the equatorial electrojet, J. Geophys. Res., 77, 2975, 1972.
Ronchi, C., R. N. Sudan, and D. T. Farley, Numerical simulations of large-scale plasma turbulence in the daytime equatorial electrojet, J. Geophys. Res., 96, 21263, 1991.

Schlegel, K., and J. P. St-Maurice, Anomolous heating of the polar $\mathrm{E}$ region by unstable plasma waves, 1, Observations, $J$. Geophys. Res., 86, 1447, 1981.

Schuster, A., The diurnal variation of terrestrial magnetism, Philos. Trans. R. Soc. London, Ser. A, 208, 163, 1908.

Simon, A., Instability of a partially ionized plasma in crossed electric and magnetic fields, Phys. Fluids, 6, 382, 1963.

Sudan, R. J., J. Akinrimisi, and D. T. Farley, Generation of smallscale irregularities in the equatorial electrojet, J. Geophys. Res., 78 1453, 1973.

Wickwar, V. B., C. Lathuillere, W. Kofman, and G. Lejeune, Elevated electron temperatures in the auroral E-layer measured with the chatanika radar, J. Geophys. Res., 86, 4721, 1981. 